\title{
Translation regulatory long non-coding RNA 1 represents a potential prognostic biomarker for colorectal cancer
}

\author{
WEIJIA WANG ${ }^{1,2}$, XIAOLONG TANG ${ }^{1}$, HUI QU $^{1}$ and QINGSI HE $^{1}$ \\ ${ }^{1}$ Department of General Surgery, Qilu Hospital of Shandong University, Jinan, Shandong 250012; \\ ${ }^{2}$ Department of General Surgery, Linyi People's Hospital, Linyi, Shandong 276000, P.R. China
}

Received September 18, 2019; Accepted March 5, 2020

DOI: 10.3892/ol.2020.11532

\begin{abstract}
Long non-coding RNAs (lncRNAs) have attracted a lot of attention for their role in the development, progression and prognosis of colorectal cancer (CRC). However, little is known on the clinical significance of the translation regulatory lncRNA 1 (TRERNA1) in CRC. The present study aimed to explore the clinical value of TRERNA1 in patients with CRC. A total of 89 cancer-associated lncRNA genes were analyzed using the RT ${ }^{2}$ IncRNA PCR array Human Cancer PathwayFinder. Following the PCR array, reverse transcription-quantitative (RT-q)PCR was conducted to identify the differential expression of TRERNA1 between 130 CRC and corresponding non-tumorous adjacent tissues. Additionally, the association between TRERNA1 expression and clinical characteristics was analyzed. Furthermore, TRERNA1 expression was knocked down via small interfering RNAs. The results of the PCR array and RT-qPCR revealed that TRERNA1 expression was significantly upregulated in CRC tissues compared with in adjacent normal tissues. TRERNA1 upregulation was positively associated with distant metastasis, perineural invasion, TNM stage, node metastasis stage and tumor diameter. Multivariate analysis revealed that patients with higher TRERNA1 expression had a shorter overall survival (OS) time and a less favorable prognosis compared with those in the low TRERNA1 expression group. Knockdown of TRERNA1 inhibited invasion and metastasis of CRC cells via regulating Snail expression. In conclusion, TRERNA1 expression was upregulated in CRC tissues. High expression levels of TRERNA1 may be associated with poor OS times, a less favorable prognosis and lymph node metastasis in patients with CRC. TRERNA1 may therefore serve as a useful and novel biomarker for CRC lymph node metastasis and prognosis.
\end{abstract}

Correspondence to: Dr Hui Qu or Dr Qingsi He, Department of General Surgery, Qilu Hospital of Shandong University, 107 West of Wenhua Street, Lixia, Jinan, Shandong 250012, P.R. China

E-mail: 201989226053@sdu.edu.cn

E-mail: doctorheqs@163.com

Key words: translation regulatory long non-coding RNA 1, long non-coding RNA, colorectal cancer, PCR array, prognosis

\section{Introduction}

Colorectal cancer (CRC) was the third most commonly diagnosed malignancy and the second leading cause of cancer-associated mortality worldwide in 2012 (1). This could be effectively reduced by early screening. It is estimated that there were 3,763,000 newly diagnosed cases of CRC and 191,000 deaths attributable to CRC in 2015 (2). There has recently been a growing interest in identifying the causes of CRC development and novel approaches to prevent CRC and improve early diagnosis and treatments. Although researchers have made great progress in revealing the underlying mechanisms of CRC development and metastasis, certain aspects remain unclear. Additionally, early diagnosis of CRC remains unsatisfactory due to metastasis, genetic heterogeneity and living habits (3). Further investigations are required to identify novel and useful biomarkers for the early diagnosis and treatment of CRC.

Long non-coding RNAs (lncRNAs) are transcripts of $>200$ nucleotides in length with limited or no coding potential (4). LncRNAs exhibit an mRNA-like structure with a poly-A tail and a promoter region. They not only assist in the intermediary delivery of genetic information, but they also serve a number of regulatory functions, including dynamic expression and differential splicing (5). They have attracted a lot of attention due to their involvement in a number of physiological processes (6). LncRNAs are involved in multiple regulatory processes, including $\mathrm{X}$-chromosome silencing, genomic imprinting, chromatin modification, transcriptional activation, transcriptional interference and intranuclear transport (7).

Several studies have revealed an association between aberrant lncRNA expression and human diseases such as cancer, neurodegenerative and cardiovascular diseases $(8,9)$. LncRNAs influence almost all of the hallmarks of cancer, including sustaining proliferative signaling, evading growth suppressors, resisting cell death, enabling replicative immortality, inducing angiogenesis, promoting invasion/metastasis, genome instability, inflammation, reprogramming of energy metabolism and evading immune destruction (10). Aberrant lncRNA expression is associated with the progression of various types of cancer, such as breast, liver, bladder, prostate, lung, gastric and CRC $(11,12)$.

Increasing evidence has demonstrated that specific lncRNAs are abnormally expressed in numerous types of 
cancer, including CRC, and function as tumor suppressor genes, oncogenes or both (13). Increasing studies have highlighted that lncRNA dysregulation serves a pivotal role in proliferation, angiogenesis, metastasis, invasion, apoptosis and genome instability in CRC, which are consequently associated with clinical implications (14-17). Colon cancer-associated transcript (CCAT) 2 was identified as a novel lncRNA that is upregulated in microsatellite-stable CRC, contributing to CRC pathogenesis such as tumor growth, metastasis and chromosomal instability (18). The long intergenic non-protein coding RNA 2598 is upregulated in CRC in a stage-dependent manner and is able to promote the migration, invasion and epithelial-to-mesenchymal transition (EMT) of CRC cells (19). Master factors of EMT, such as Snail, play important role in CRC $(20,21)$. Snail is a conserved zinc-finger transcription factor, which can induce EMT in a variety of tissues, and different EMT programs might exist $(22,23)$. Lnc-GNAT1-1 is expressed at low levels in CRC and functions as a tumor suppressor by regulating RKIP (Raf kinase inhibitor protein)-NF- $\kappa B-S n a i l$ circuit (24). The expression of metastasis-associated lung adenocarcinoma transcript 1 (MALAT1) is associated with CRC metastasis, while decreased expression of MALAT1 inhibits nuclear translocation of $\beta$-catenin and attenuates $\mathrm{Wnt} / \beta$-catenin signaling, resulting in decreased CRC invasion and metastasis (25-27). Furthermore, lncRNAs with altered expression levels in CRC tissues may serve as potential prognostic biomarkers and therapeutic targets $(28,29)$. Although a total of 556 upregulated and 1,040 downregulated lncRNAs have been identified in CRC tissues, the underlying mechanisms of each lncRNA in CRC remain unclear (30).

Therefore, the present study investigated the expression profile of lncRNAs in CRC and paired normal tissues using a PCR array to assess the association between abnormally expressed lncRNAs and CRC, and to identify potential prognostic biomarkers for CRC.

\section{Materials and methods}

Clinical samples. Three patients (two females aged 50 and 47 years and one male aged 48 years) with CRC were randomly recruited from the Qilu Hospital of Shandong University (Jinan, China) for $\mathrm{RT}^{2}$ IncRNA PCR array Human Cancer PathwayFinder (Qiagen, GmbH). A total of 130 CRC and paired adjacent normal tissues were collected from patients. The patient cohort consisted of 96 males and 34 females; 72 people were $>60$ years old and 58 people were $\leq 60$ years old who underwent surgical resection at the Department of General Surgery at Qilu Hospital of Shandong University between April 2013 and December 2014 in order to validate the results of the PCR array. All patients had pathologically confirmed colorectal adenocarcinoma. Tumors were staged according to the TNM staging system of the American Joint Committee on Cancer (7th edition) (31). None of the patients had received radiotherapy or chemotherapy prior to surgical resection. All isolated samples were frozen in liquid nitrogen and then stored at $-80^{\circ} \mathrm{C}$ prior to RNA extraction. The experimental protocol of the present study was approved by the Ethics Committee of Qilu Hospital of Shandong University. All tissues were removed by the same method and the same inclusion criteria were used as in the bigger patient cohort. The distance between normal tissue and cancerous tissue $>5 \mathrm{~cm}$, and the normal mucosa had no visible deformation. Written informed consent was provided by all participants.

PCR array. Gene expression was measured using the $\mathrm{RT}^{2}$ IncRNA PCR array Human Cancer PathwayFinder (Qiagen, $\mathrm{GmbH}$ ) (32). Mature RNA was isolated from tissues using an RNA extraction kit (cat. no. 74106; Qiagen $\mathrm{GmbH}$ ) according to the manufacturer's protocol. The RNA quality was determined using a spectrophotometer and the RNA was subsequently reverse transcribed using $\mathrm{RT}^{2}$ First Stand kit (cat no. 330401; Qiagen $\mathrm{GmbH}$ ) according to the manufacturer's protocol with steps at $42^{\circ} \mathrm{C}$ for 5 min to remove genomic DNA, ice bath for at least $1 \mathrm{~min}, 42^{\circ} \mathrm{C}$ for $15 \mathrm{~min}$ to perform reverse transcription and $95^{\circ} \mathrm{C}$ for $5 \mathrm{~min}$ to interrupt the reaction. The cDNA was used for the RT ${ }^{2}$ lncRNA PCR array (Qiagen, $\mathrm{GmbH}$ ) in combination with $\mathrm{RT}^{2} \mathrm{SYBR}^{\circledR}$ Green qPCR Mastermix (Qiagen, GmbH). A total of 89 lncRNA genes associated with CRC were assessed on three paired samples using the $\mathrm{RT}^{2}$ IncRNA PCR array. After qPCR, CT values were exported to an Excel file to create a table of $\mathrm{C}_{\mathrm{T}}$ values. This table was then uploaded on to the data analysis web portal at http://www.qiagen.com/geneglobe. Samples were assigned to control and test groups. $\mathrm{C}_{\mathrm{T}}$ values were normalized based on a/an Automatic selection from full panel of reference genes. The data analysis web portal calculates fold change/regulation using $\Delta \Delta \mathrm{C}_{\mathrm{t}}$ method, in which $\Delta \mathrm{Ct}$ is calculated between gene of interest (GOI) and an average of reference genes (HKG), followed by $\Delta \Delta \mathrm{C}_{\mathrm{t}}$ calculations $\left[\Delta \mathrm{C}_{\mathrm{t}}\right.$ (Test Group)- $\Delta \mathrm{C}_{\mathrm{t}}$ (Control Group)]. Fold Change is then calculated using $2^{-\Delta \Delta \mathrm{Ct}}$ formula. The data analysis web portal also plots scatter plot, volcano plot, clustergram, and heat map. This data analysis report was exported from the QIAGEN web portal at GeneGlobe. Fold change was calculated as the normalized lncRNA expression in the cancer tissue samples divided by the normalized expression in the paired normal tissue samples. $\mathrm{P}<0.05$ (two-tailed) was considered to indicate a statistically significant difference.

Reverse transcription-quantitative (RT-q)PCR analysis. Total RNA was extracted from 130 patient tissues using an RNeasy Mini kit (Qiagen, $\mathrm{GmbH}$ ). Reverse transcription was performed using the First Strand kit (Qiagen, $\mathrm{GmbH}$ ) with $1 \mu \mathrm{g}$ of RNA per sample according to the manufacturer's protocol. qPCR reactions were run on a LC96 thermal cycler (Roche Diagnostics) with an initial activation step at $95^{\circ} \mathrm{C}$ for $15 \mathrm{sec}$, followed by 40 cycles at $95^{\circ} \mathrm{C}$ for $15 \mathrm{sec}$ and $60^{\circ} \mathrm{C}$ for $1 \mathrm{~min}$. The primers for TRERNA1 PCR were purchased from Qiagen, $\mathrm{GmbH}$. Detection was repeated in triplicates. Primer sequences were as follows: Snail forward, 5'-CCTCGCTGCCAATGCTCA TCTG-3' and reverse, 5'-GCTCTGCCACCCTGGGACTC-3'; and GAPDH forward, 5'-TGACTTCAACAGCGACACCCA-3' and reverse, 5'-CACCCTGTTGCTGTAGCCAAA-3'. The fluorophore was $\mathrm{RT}^{2}$ SYBR Green qPCR Mastermix (Qiagen, $\mathrm{GmbH})$. Comparative quantification was assessed using the $2^{-\Delta \Delta \mathrm{CT}}$ method using GAPDH as the endogenous control (33).

Cell culture. The normal human colorectal FHC cell line and the human CRC HCT-8, DLD-1, SW480, HCT-116 and SW620 cell lines were purchased from the Cell Resource Center at the Shanghai Institute of Biochemistry and Cell Biology at the Chinese Academy of Sciences. HCT-8, DLD-1 and FHC cells were cultured in RPMI-1640 medium (Gibco; Thermo Fisher 
Scientific, Inc.) supplemented with $10 \%$ (v/v) FBS (Gibco; Thermo Fisher Scientific, Inc.). HCT-116 and SW480 were cultured in DMEM (Gibco; Thermo Fisher Scientific, Inc.) supplemented with $10 \%$ FBS. All cell lines were incubated in a humidified atmosphere at $37^{\circ} \mathrm{C}$ with $5 \% \mathrm{CO}_{2}$.

Small interfering (si)RNA-mediated knockdown of TRERNA1. SiRNAs were purchased from Suzhou Ribo Life Science Co., Ltd. HCT-8 and HCT-116 cells were transfected with 5 nmol siRNAs with jetPRIME ${ }^{\circledR}$ transfection reagent (Polyplus-transfection SA) when the cell density was $1 \times 10^{6}$ cells in a 6 -well plate according to the manufacturer's protocol. Subsequent experiments were performed $48 \mathrm{~h}$ after transfection. The siRNAs sequences were as follows:

Si-negative control (Si-NC), 5'-UUCUUCGAAACGUGUCAC GUT-3'; and Si-TRERNA1, 5'-GAAGGGAACCAGUGCUAA AUU-3'.

Transwell assay. Matrigel (cat. no. 356234; Corning Life Sciences) and serum-free medium were mixed in a 1:4 ratio (v/v) to create a mixed liquid. A total of $20 \mu 1$ mixed liquid was added to Transwell chambers (cat. no. 3422; Corning Life Sciences) inserted in 24-well plates, which were incubated for $3 \mathrm{~h}$ at $37^{\circ} \mathrm{C}$ to speed up the solidification of Matrigel. SiRNA-transfected HCT-8 or HCT-116 cells were counted, and $5 \times 10^{4}$ cells/well were plated into the upper chambers in serum-free medium (HCT- 8 cells were incubated in RPMI-1640 medium; HCT-116 cells were incubated in DMEM medium). A total of $600 \mu 1$ medium (HCT-8 cells were incubated in RPMI-1640 medium; HCT-116 were incubated in DMEM medium) supplemented with $20 \%$ (v/v) FBS was added in the lower chambers. After $18 \mathrm{~h}$ of incubation at $37^{\circ} \mathrm{C}$, the cells and Matrigel in the upper chambers were removed using cotton swabs, and the cells in the lower chambers were fixed with anhydrous methanol at room temperature for 15 min and stained with $0.05 \%$ crystal violet staining solution (Beijing Solarbio Science \& Technology Co., Ltd.) at room temperature for $15 \mathrm{~min}$. Finally, the cells were imaged using a light microscope (magnification, x100) and counted to compare cell invasion between the control and transfected groups. The same steps without the addition of Matrigel were followed to analyze cell migration.

Western blot analysis. HCT-8 and HCT-116 cells were lysed using the high RIPA lysis buffer with proteinase inhibitor PMSF (Beijing Solarbio Science \& Technology Co., Ltd.) at a ratio of 100:1 (v/v), after transfection for $48 \mathrm{~h}$. The protein concentration was determined using the bicinchoninic acid reagent kit (Thermo Fisher Scientific, Inc.) according to the manufacturer's protocol. A total of $30 \mu \mathrm{g}$ of protein was loaded per lane and separated using a 10\% SDS-PAGE gel and subsequently transferred onto PVDF membranes (EMD Millipore). The membranes were blocked using 5\% skimmed milk for $1 \mathrm{~h}$ at room temperature, followed by overnight incubation with primary antibodies against Snail (1:1,000; cat. no. 3879; Cell Signaling Technology, Inc.), E-cadherin (1:1,000; cat no. 3195; Cell Signaling Technology, Inc.) and $\beta$-actin $(1: 1,000$; cat no. 3195; Cell Signaling Technology, Inc. $x)$ at $4^{\circ} \mathrm{C}$. The membranes were subsequently incubated with anti-rabbit
Table I. Fold-change of long non-coding RNAs detected via PCR array.

\begin{tabular}{llcc}
\hline Position & Gene symbol & Fold change & P-value \\
\hline A05 & BANCR & 21.14 & 0.045 \\
G05 & TRERNA1 & 21.88 & 0.005 \\
\hline
\end{tabular}

$\mathrm{P}<0.05$. BANCR, BRAF-activated non-protein coding RNA; TRERNA1, translation regulatory long non-coding RNA 1.

horseradish peroxidase-labeled secondary antibodies (1:5,000; cat. no. SA00001-2; Wuhan Sanying Biotechnology) for $1 \mathrm{~h}$ at room temperature. Finally, the PVDF membranes were developed using the enhanced chemiluminescence method using Luminata ${ }^{\mathrm{TM}}$ Western HRP Substrates (EMD Millipore) and detected using a chemiluminometer (Tanon Science and Technology Co., Ltd.).

Prediction of lncRNA/mRNA and miRNA interactions. MiRanda (v1.0b) was used to predicted the miRNAs binding both TRERNA1 and Snail according to Anton's study (34). A hit between any predicted miRNA and a target lncRNA/mRNA was considered with a score of 100 or higher, corresponding to at least a perfect seed match.

Statistical analysis. Statistical analysis was conducted using SPSS v25.0 (IBM Corp.). All data was presented as mean \pm SD. Comparisons between two groups were performed by Student's unpaired t-test, and categorical data were analyzed using Fisher's exact test. A paired t-test was used to analyze the differential expression of TRERNA1 in cancer tissues compared with adjacent normal tissues. If variance tested by one-way ANOVA was statistically significant, Tukey's multiple comparisons test was used to make comparisons among multiple groups. Overall survival between experimental groups were compared by a log-rank (Mantel-Cox) test in the Kaplan-Meier analysis. Spearman's correlation coefficient analysis was used to assess the correlation between TRERNA1 and Snail levels in CRC tissues. $\mathrm{P}<0.05$ (two-tailed) was considered to indicate a statistically significant difference.

\section{Results}

Differentially expressed lncRNAs in CRC tissues. To test the effect of lncRNAs in CRC tissues, three pairs of CRC and non-tumorous adjacent tissues were selected. RT ${ }^{2}$ lncRNA PCR array Human Cancer PathwayFinder was used to simultaneously monitor the expression levels of 89 lncRNAs that resulted associated with tumorigenesis and CRC by relative RT-qPCR. More information regarding lncRNA genes is listed in Table SI. The differential expression of the 89 lncRNAs in CRC tissues compared with non-tumorous adjacent tissues is displayed in Fig. 1A. The volcano plots in Fig. 1B indicate significant changes in gene expression. The expression levels of 4 lncRNAs were downregulated in CRC tissues (fold change $>2$ ), while 75 lncRNAs were upregulated in CRC tissues compared with non-tumorous 
A

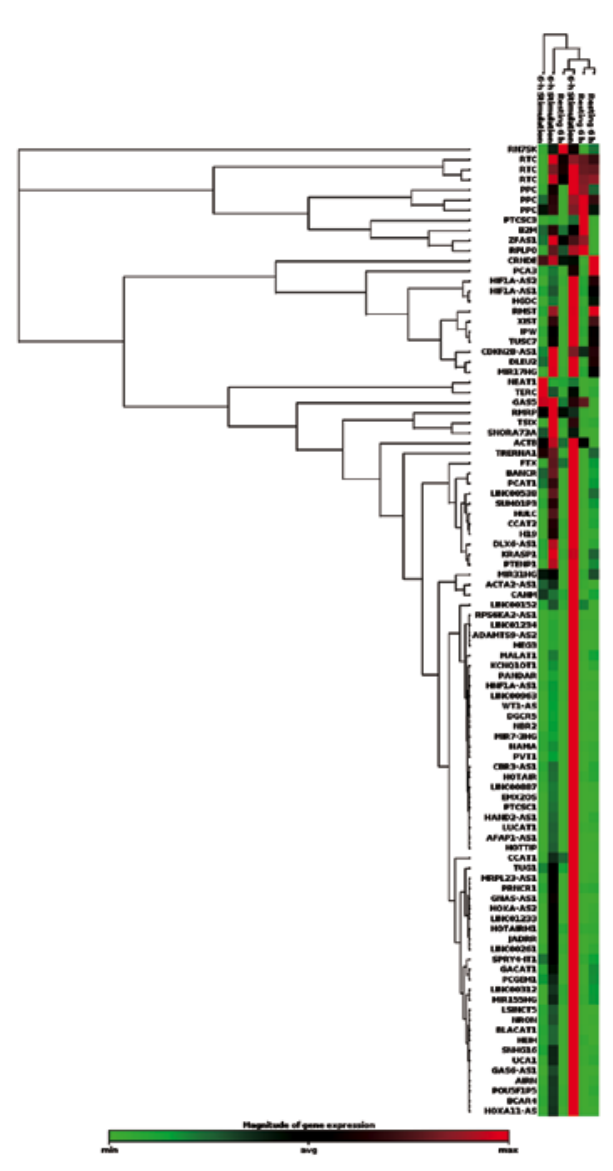

B

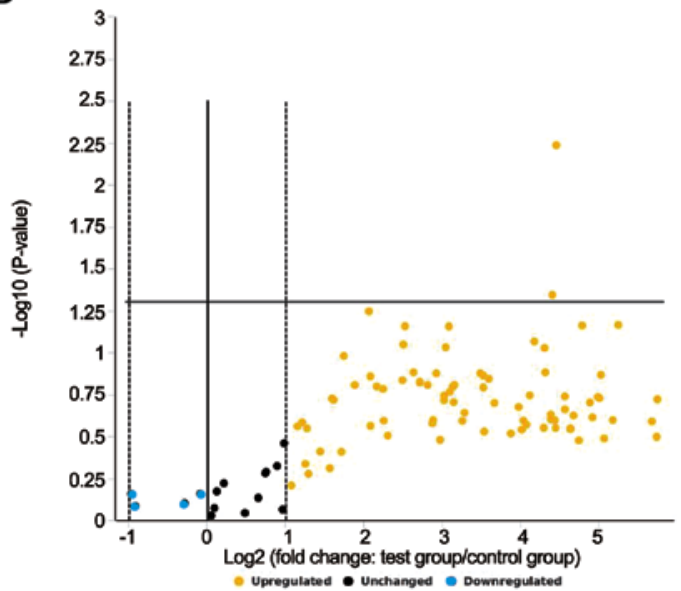

C

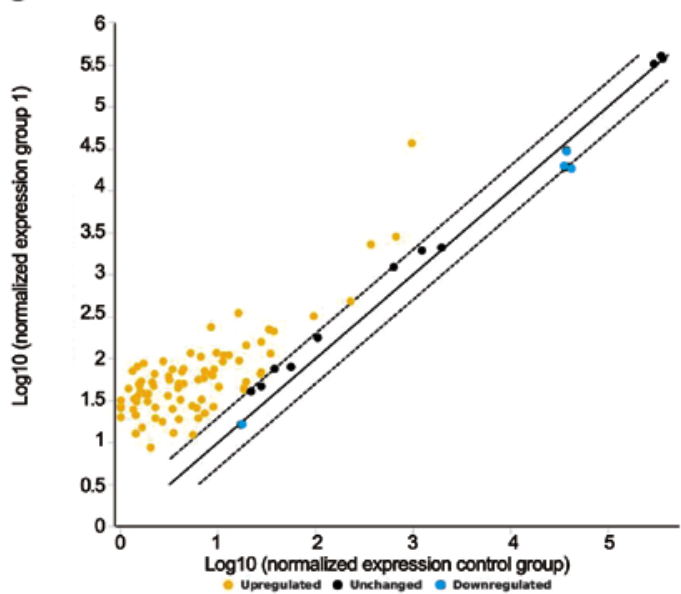

Figure 1. Differential expression of cancer-associated lncRNAs in CRC. (A) Heat map of the expression levels of 89 lncRNAs in CRC tissues and corresponding non-tumorous adjacent tissues $(n=3)$. Green to red represents lncRNA expression from low to high, respectively. (B) Volcano plot identifying significant gene expression changes. $(\mathrm{P}<0.05)$. Yellow dots represent upregulated lncRNAs; black dots represent unchanged lncRNAs; and blue dots represent downregulated IncRNAs. (C) Scatter plot comparing the normalized expression of every gene in the array between the control group and test group. Yellow dots represent upregulated lncRNAs; black dots represent unchanged lncRNAs; blue dots represent downregulated lncRNAs. CRC, colorectal cancer; lncRNA, long non-coding RNA.

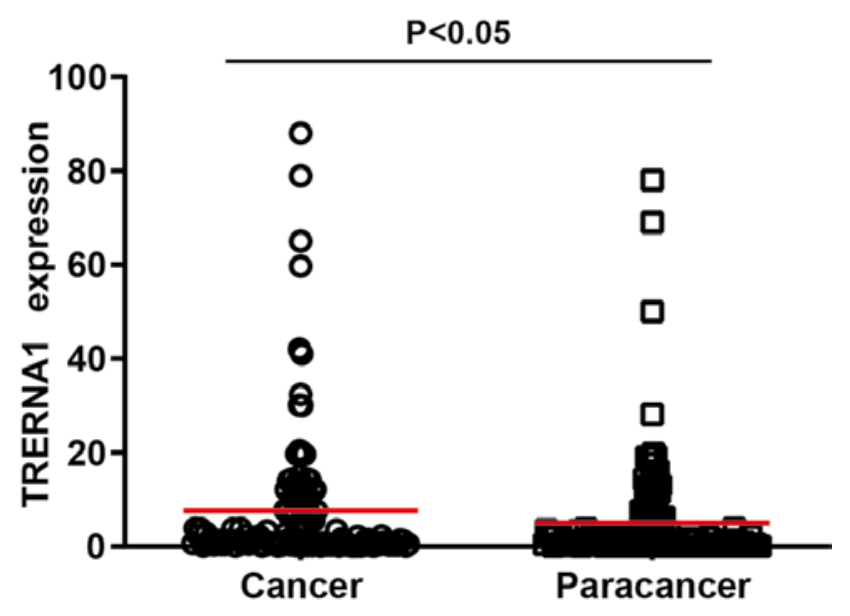

Figure 2. Reverse transcription-quantitative PCR data indicating that TRERNA1 is upregulated in colorectal cancer tissues compared with paracancer tissues. TRERNA1, translation regulatory long non-coding RNA 1.

adjacent tissues (fold change $>2$ ). A total of 10 lncRNAs exhibited no significant changes (fold change $<2$ ). The scatter plot analysis compared the normalized expression levels of each gene on the array between the control group and test group by plotting them against one another, to visualize large gene expression changes. Changes in lncRNA expression were observed between tumor and non-tumorous adjacent tissues (Fig. 1C). Additionally, 2/89 lncRNAs were significantly upregulated $(\mathrm{P}<0.05)$, namely TRERNA1 and BRAF-activated non-protein coding RNA (BANCR), with fold changes of 21.88 and 21.14 , respectively (Table I). It has been demonstrated that TRERNA1 is upregulated in gastric carcinoma and hepatic carcinoma, and that it promotes cell migration and invasion in gastric cancer and hepatic carcinoma (35-37). However, little is known about the role of TRERNA1 in CRC. Therefore, considering the results of PCR array TRERNA1 was selected for follow-up experiments.

TRERNAl expression is upregulated in CRC tissues. To assess the effect of TRERNA1 in CRC, TRERNA1 expression was analyzed in $130 \mathrm{CRC}$ and non-tumorous adjacent tissues by RT-qPCR. The results revealed that TRERNA1 expression was upregulated in $\mathrm{CRC}$ tissues compared with non-tumorous 


\section{A}

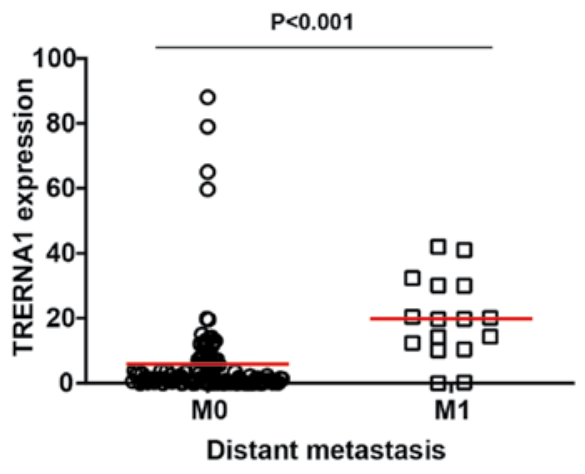

C

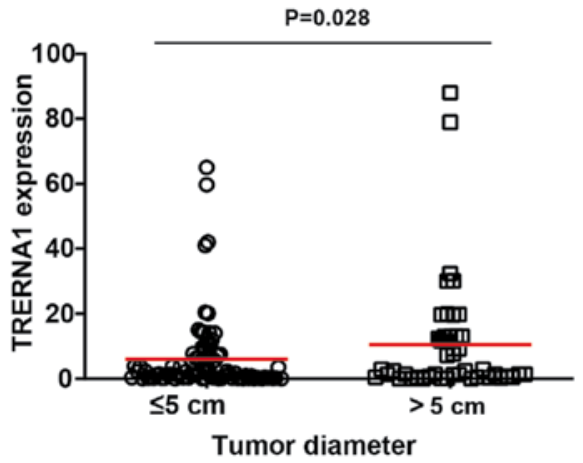

E

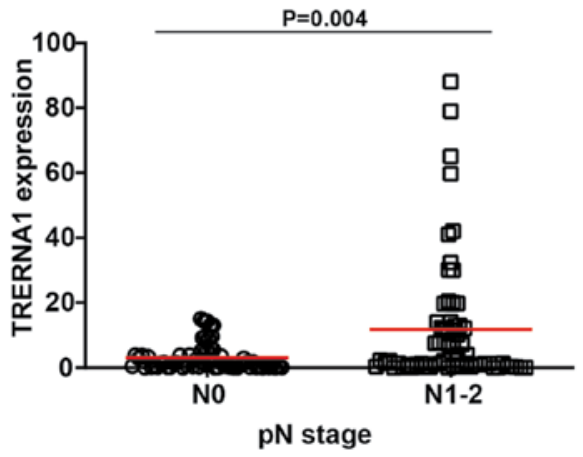

$\mathrm{B}$
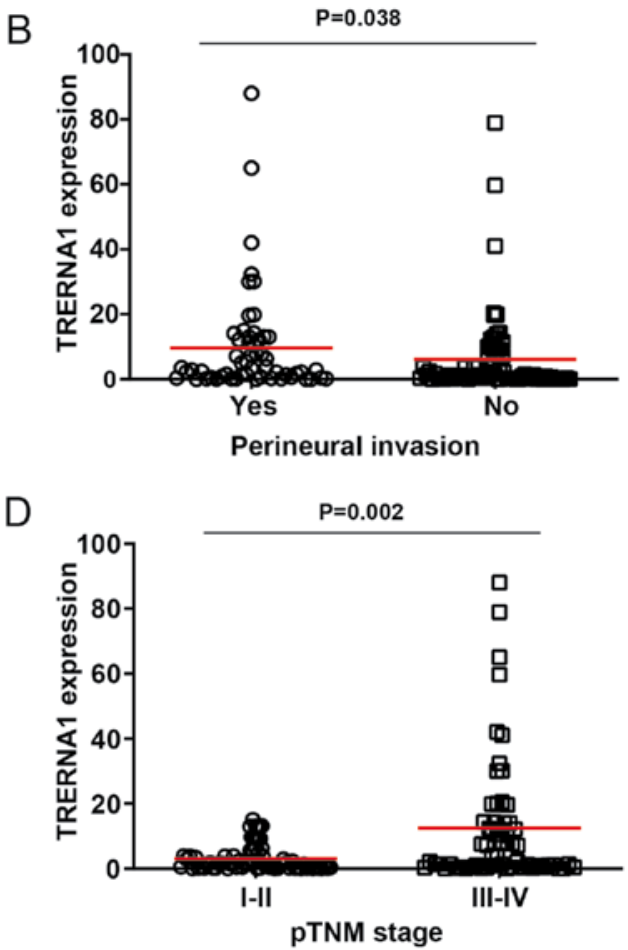

Figure 3. Association between TRERNA1 expression and clinicopathological parameters of patients with colorectal cancer. TRERNA1 expression was associated with (A) distant metastasis, (B) perineural invasion, (C) tumor diameter and (D) pTNM stage. (E) TRERNA1 expression was significantly higher in patients with N1-2 stage compared with N0 stage. TRERNA1, translation regulatory long non-coding RNA 1; pTNM, pathological TNM stage.

adjacent tissues (Fig. 2). In addition, the association between TRERNA1 expression and the clinicopathological parameters of patients with CRC was analyzed. The results suggested that TRERNA1 expression was associated with distant metastasis, perineural invasion, TNM stage, node metastasis stage and tumor diameter, but not with age, sex, tumor location and differentiation (Fig. 3A-D; Table II). For example, the expression levels of TRERNA1 in patients with M1 stage were significantly higher than in those with M0 stage (Table II). According to pathological stage (pTNM), patients with stage III-IV exhibited higher expression levels of TRERNA1 compared with patients with stage I-II (Fig. 3D). Next, the effect of TRERNA1 on pathological node metastasis stage was analyzed $(\mathrm{pN})$, which revealed that patients with stage I-II exhibited higher expression levels of TRERNA1 compared with patients with stage 0 ( Fig. 3E).

Prognostic potential of TRERNAl in CRC. All 130 patients included in the present study were classified into two sub-groups based on the expression levels of TRERNA1.

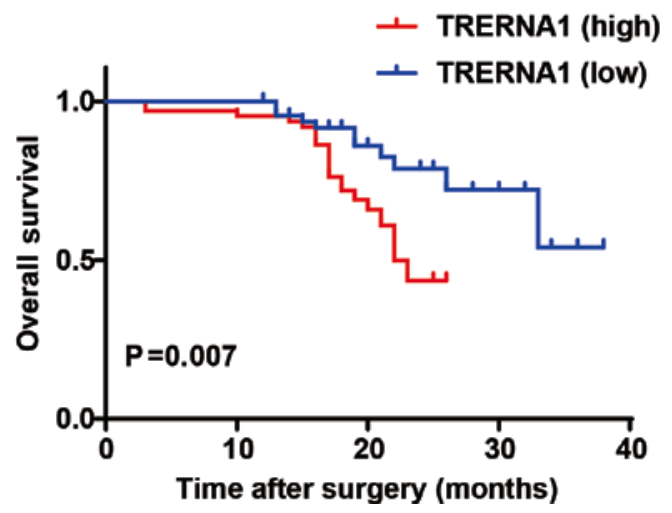

Figure 4. TRERNA1 expression is associated with patient's postoperative OS time. Patients with colorectal cancer with high TRERNA1 expression had shorter OS times than those with low TRERNA1 expression. OS, overall survival; TRERNA1, translation regulatory long non-coding RNA 1.

Patients with TRERNA1 expression levels higher than the median value 1.55 were assigned to the high expression group, 
Table II. Association between TRERNA1 expression and the clinicopathological parameters of patients with colorectal cancer $(n=130)$.

\begin{tabular}{|c|c|c|c|c|}
\hline Variable & Patients, $\mathrm{n}$ & $\begin{array}{l}\text { TRERNA1 expression level, } \\
\text { median }(95 \% \mathrm{CI})\end{array}$ & Z-value & P-value \\
\hline Sex & & & -0.32 & 0.753 \\
\hline Male & 96 & $1.61(0.35-9.63)$ & & \\
\hline Female & 34 & $1.38(0.49-7.59)$ & & \\
\hline Age, years & & & 1.18 & 0.239 \\
\hline$\leq 60$ & 58 & $2.43(0.99-7.76)$ & & \\
\hline$>60$ & 72 & $1.38(0.31-9.63)$ & & \\
\hline Tumor location & & & 1.68 & 0.093 \\
\hline Colon & 62 & $1.475(0.25-6.93)$ & & \\
\hline Rectum & 68 & $2.43(0.5-12.94)$ & & \\
\hline Tumor diameter, $\mathrm{cm}$ & & & 2.20 & $0.028^{\mathrm{a}}$ \\
\hline$\leq 5$ & 84 & $1.02(0.19-7.76)$ & & \\
\hline$>5$ & 46 & $2.89(0.74-10.42)$ & & \\
\hline Tumor differentiation & & & -0.56 & 0.575 \\
\hline Poor & 30 & $1.35(0.21-10.16)$ & & \\
\hline Well/moderate & 100 & $1.95(0.39-9.4)$ & & \\
\hline pT stage & & & 0.97 & 0.332 \\
\hline $\mathrm{T} 1-3$ & 98 & $1.54(0.37-7.76)$ & & \\
\hline $\mathrm{T} 4$ & 32 & $1.89(0.44-13.56)$ & & \\
\hline pN stage & & & -2.92 & $0.004^{\mathrm{a}}$ \\
\hline No & 62 & $1.38(0.21-3.83)$ & & \\
\hline $\mathrm{N} 1-2$ & 68 & $3.18(0.86-13.50)$ & & \\
\hline Distant metastasis (M stage) & & & 4.33 & $<0.001^{\mathrm{a}}$ \\
\hline M0 & 114 & $1.36(0.37-6.27)$ & & \\
\hline M1 & 16 & $19.83(11.38-30.03)$ & & \\
\hline pTNM stage & & & 3.06 & $0.002^{\mathrm{a}}$ \\
\hline I-II & 66 & $1.38(0.21-3.81)$ & & \\
\hline III-IV & 64 & $5.83(0.63-14.31)$ & & \\
\hline Lymphovascular invasion & & & -0.57 & 0.567 \\
\hline Yes & 10 & $1.36(0.39-5.57)$ & & \\
\hline No & 120 & $1.61(0.38-9.63)$ & & \\
\hline Perineural invasion & & & 2.08 & $0.038^{\mathrm{a}}$ \\
\hline Yes & 56 & $3.15(0.57-12.52)$ & & \\
\hline No & 74 & $1.26(0.33-7.59)$ & & \\
\hline
\end{tabular}

${ }^{\mathrm{a}} \mathrm{P}<0.05$. TRERNA1, translation regulatory long non-coding RNA 1; pTNM, pathological TNM.

while patients with TRERNA1 expression levels lower than the median value were assigned to the low expression group. The term overall survival (OS) time was used to describe the chance of survival. The association between TRERNA1 expression and OS time in patients with CRC was investigated via Kaplan-Meier analysis. The results of the survival curve revealed that patients with CRC with high TRERNA1 expression had shorter OS times than those with low TRERNA1 expression (Fig. 4). In addition, the multivariate analysis revealed that high TRERNA1 expression, distant metastasis and patient node metastasis were associated with a less favorable prognosis in patients with CRC (Table III). The results of the present study revealed that TRERNA1 may serve as a prognostic marker for patients with CRC.

siRNA-mediated knockdown of TRERNAl inhibits invasion and migration of CRC cells. Based on results from clinical pathology analyses and previous studies $(35,36)$, it was hypothesized that TRERNA1 may affect the invasion and migration abilities of CRC cells. Therefore, TRERNA1 expression in the normal human colorectal FHC cell line and five CRC cell lines was detected. The results revealed that TRERNA1 expression was upregulated in HCT-8, DLD-1, SW480 and HCT-116 cell lines compared with in FHC cells (Fig. 5A). Subsequently, the 
Table III. Univariate and multivariate overall survival analysis of clinicopathological factors.

\begin{tabular}{|c|c|c|c|c|}
\hline \multirow[b]{2}{*}{ Variable } & \multicolumn{2}{|c|}{ Univariate analysis } & \multicolumn{2}{|c|}{ Multivariate analysis } \\
\hline & $\mathrm{HR}(95 \% \mathrm{CI})$ & P-value & $\mathrm{HR}(95 \% \mathrm{CI})$ & P-value \\
\hline Sex (male vs. female) & $0.86(0.37-1.99)$ & 0.727 & & \\
\hline Age, years ( $\leq 60$ vs. $>60)$ & $0.77(0.38-1.55)$ & 0.461 & & \\
\hline Tumor diameter, $\mathrm{cm}(\leq 5 \mathrm{vs} .>5)$ & $1.01(0.51-2.03)$ & 0.968 & & \\
\hline Tumor location (colon vs. rectum) & $2.31(1.12-4.73)$ & 0.023 & & \\
\hline Differentiation (well/moderate vs. poor) & $0.71(0.34-1.51)$ & 0.374 & & \\
\hline pT stage (T1-3 vs. T4) & $2.34(1.16-4.72)$ & 0.017 & & \\
\hline pN stage (N0 vs. N1-2) & $3.85(1.58-9.37)$ & 0.003 & & \\
\hline Distant metastasis (M0 vs. M1) & $3.97(1.90-8.28)$ & $<0.001$ & $2.51(1.10-5.71)$ & $0.029^{\mathrm{a}}$ \\
\hline pTNM stage (I-II vs. III-IV) & $7.99(2.80-22.82)$ & $<0.001$ & $6.24(2.10-18.58)$ & $0.001^{\mathrm{a}}$ \\
\hline Lymphovascular invasion (no vs. yes) & $1.43(0.54-3.84)$ & 0.474 & & \\
\hline Perineural invasion (no vs. yes) & $1.20(0.59-2.41)$ & 0.616 & & \\
\hline TRERNA1 expression (low vs. high) & $2.76(1.27-5.97)$ & 0.010 & $2.68(1.25-5.73)$ & $0.011^{\mathrm{a}}$ \\
\hline
\end{tabular}

${ }^{\text {a }} \mathrm{P}<0.05$. TRERNA1, translation regulatory long non-coding RNA 1; HR, hazard ratio; CI, confidence interval.

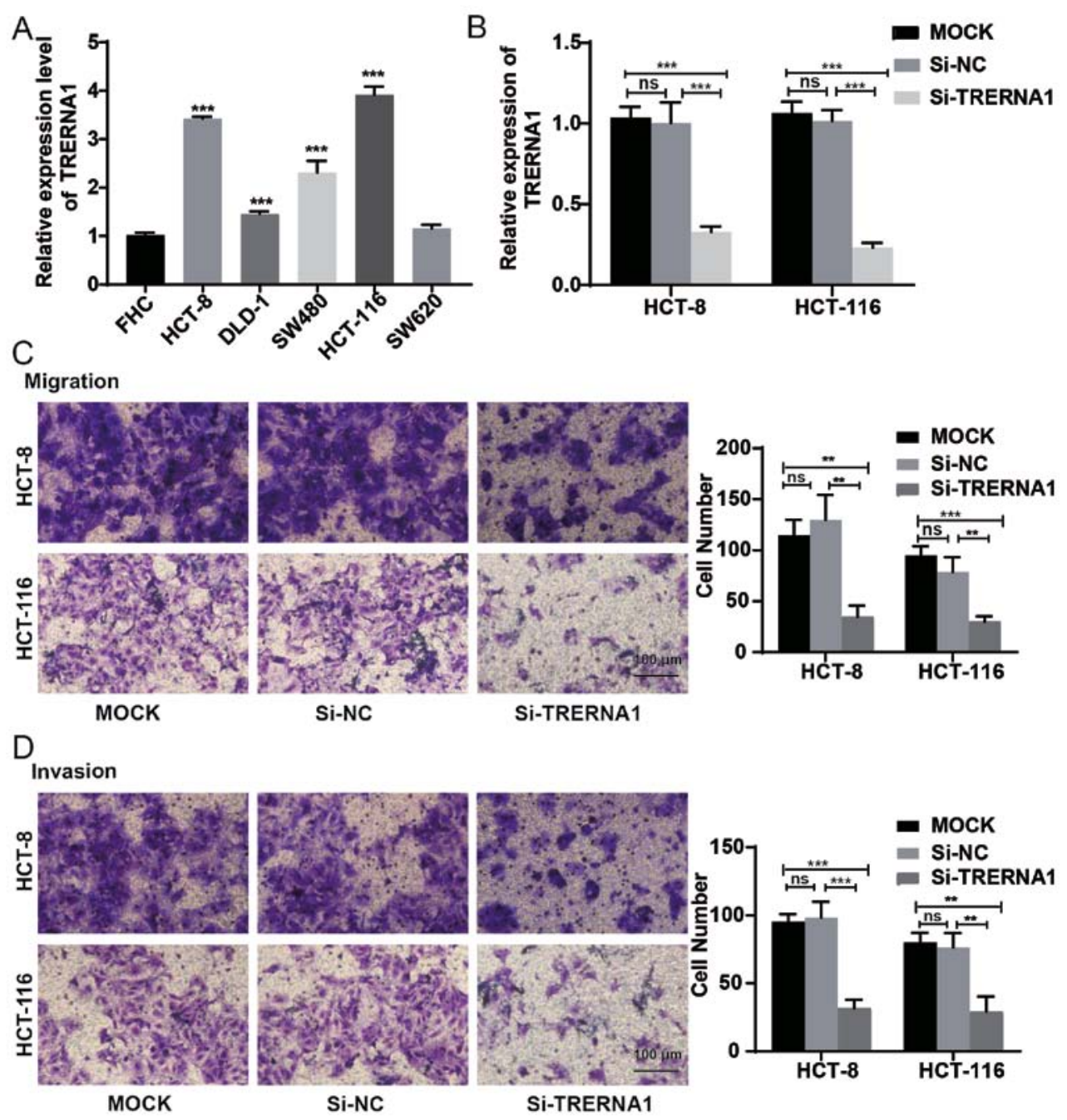

Figure 5. TRERNA1 knockdown inhibits the invasion and metastasis of CRC cells. (A) TRERNA1 expression was analyzed in the normal human colorectal FHC cell line and five CRC cell lines. (B) Reverse transcription-quantitative PCR analysis showing that Si-TRERNA1 significantly decreased TRERNA1 expression in HCT-8 and HCT-116 cells. (C) Migration assays indicate that TRERNA1 knockdown inhibited the migratory abilities of HCT-8 and HCT-116 cells. Scale bar $=100 \mu \mathrm{m}$. (D) Invasion assays reveal that TRERNA1 knockdown inhibited the invasive abilities of HCT-8 and HCT-116 cells. Scale bar $=100 \mu \mathrm{m}{ }^{* *} \mathrm{P}<0.01$; ${ }^{* * * *} \mathrm{P}<0.001$. TRERNA1, translation regulatory long non-coding RNA 1; CRC, colorectal cancer; Si, small interfering; NC, negative control; ns, not significant. 

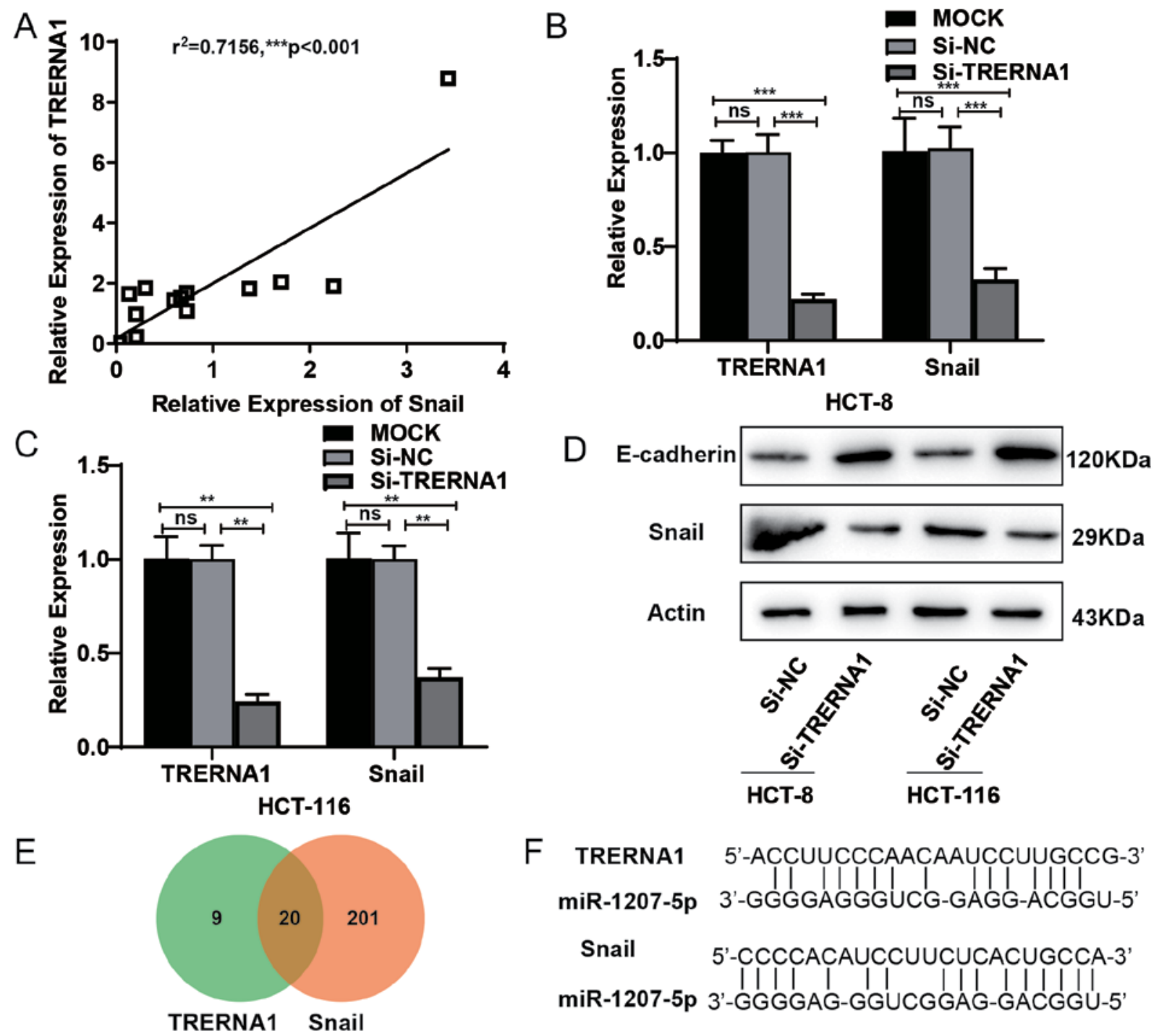

Figure 6. TRERNA1 knockdown suppresses Snail expression. (A) RT-qPCR analysis revealed that TRERNA1 expression was positively correlated with Snail expression in colorectal cancer tissues $\left(\mathrm{r}^{2}=0.7156 ; \mathrm{P}<0.001\right)$. RT-qPCR analysis indicating that TRERNA1 knockdown inhibited Snail expression in (B) HCT-8 and (C) HCT-116 cells. (D) Western blot results revealed that TRERNA1 knockdown resulted in the downregulation of Snail expression and upregulation of E-cadherin expression. (E) Bioinformatics analysis of miRNAs bound by TRERNA1 and Snail; a total of 29 miRNAs were bound by TRERNA1 (green) and 221 miRNAs were bound by Snail (orange). (F) Binding sequences of TRERNA1 and Snail to miR-1207-5p. ${ }^{* *} \mathrm{P}<0.01$; $^{* * *} \mathrm{P}<0.001$. RT-qPCR, reverse transcription-quantitative PCR; TRERNA1, translation regulatory long non-coding RNA 1; miRNA/miR, microRNA; Si, small interfering; NC, negative control.

human CRC HCT-8 and HCT-116 cell lines were used to detect the effect of TRERNA1 on the invasion and migration abilities of CRC cells in vitro. TRERNA1 expression was knocked down with specific siRNA sequences, and the results are presented in Fig. 5B. Following TRERNA1 knockdown, the number of HCT- 8 and HCT-116 cells that migrated or invaded to the other side of the membrane was significantly decreased in the experimental groups compared with the control groups (Fig. 5C and D). This suggests that the downregulation of TRERNA1 expression inhibits the invasion and migration abilities of CRC cells.

Knockdown of TRERNA1 suppresses Snail expression. The present study revealed that high TRERNA1 expression was associated with CRC metastasis and that the invasion and migratory abilities of CRC cells were inhibited following TRERNA1 knockdown. Additionally, previous studies demonstrated that TRERNA1 regulates Snail expression as an enhancer of SNAI1 promoter (35). Results from the present study revealed that TRERNA1 expression was positively correlated with Snail expression in CRC tissues (Fig. 6A). TRERNA1 knockdown suppressed the expression levels of Snail mRNA (Fig. 6B and C). Western blot results revealed that following TRERNA1 knockdown, Snail expression was downregulated, while E-cadherin expression was upregulated (Fig. 6D). These results suggested that TRERNA1 may affect EMT in CRC metastasis via regulation of Snail expression. A total of 20 microRNAs (miRs) were predicted to bind both TRERNA1 and Snail, using miRanda v1.0b (energy <-100; Fig. 6E; Tables SII-SIV). MiR-1207-5p was of particular interest, since studies have demonstrated that miR-1207-5p is expressed at low levels in CRC $(38,39)$. It was therefore speculated that TRERNA1 may regulate miR-1207-5p and Snail expression via endogenous competition (Fig. 6F). 


\section{Discussion}

Numerous genetic factors influence the pathogenesis, development, metastasis and prognosis of CRC, including IncRNAs (29). The present study revealed two differentially expressed IncRNAs, TRERNA1 and BANCR, in CRC samples compared with adjacent normal tissues, via PCR array. It has been previously reported that BANCR regulates the microRNA (miR)-203/chromosome segregation 1 like axis and increases chemosensitization of CRC cells to Adriamycin, and BANCR upregulation is associated with lymph node metastasis and a poor prognosis in patients with CRC $(28,40,41)$. It has been previously demonstrated that upregulation of BANCR is associated with lymph node metastasis and a poor prognosis in patients with CRC (17). However, there are a few studies on the role of TRERNA1 in CRC. The findings from the present study revealed that high TRERNA1 expression in CRC tissues was negatively associated with survival time and acted as an independent prognostic factor for patients with CRC.

LncRNAs, which are $>200$ nucleotides in length, have different functions in various cell processes and diseases. Numerous lncRNAs are abnormally expressed in CRC (42). CCAT1 was the first identified lncRNA with potential as a diagnostic marker in CRC and tumor-associated tissues (43). A total of $>2,300$ lncRNAs were dysregulated among 33,045 lncRNAs tested at the genome-wide level in CRC (44), among which TRERNA1was not included.

TRERNA1 is an EMT master regulator transcription factor that has been demonstrated to serve an important role in the invasion and metastasis of gastric cancer $(35,36)$. Additionally, TRERNA1 is associated with invasion and a poor response to chemotherapy in chronic lymphocytic leukemia (45). The $\mathrm{RT}^{2}$ lncRNA PCR array is a highly sensitive gene expression profiling tool used for analyzing focused panels of cancer-associated genes (46). From the PCR array results, the top candidates were selected according to the established criteria $(>15$-fold change and $\mathrm{P}<0.05)$ and RT-qPCR was performed to investigate the expression levels of TRERNA1 in CRC tissues. The results revealed that TRERNA1 expression was significantly increased in the remaining 130 cancerous tissues compared with paired adjacent normal tissues, in accordance with the study conducted by Kim et al (44), which suggested that elevated TRERNA1 expression may be a common feature in tumor progression and metastasis.

Snail is a zinc finger transcription factor that inhibits E-cadherin transcription. E-cadherin downregulation is associated with EMT, a process that occurs during embryonic development and in invasive cancer cells (47-49). In the present study, TRERNA1 regulated Snail expression and further affected E-cadherin expression. It was speculated that TRENRNA1 may affect CRC metastasis by regulating Snail, which is consistent with the in vitro results of functional experiments conducted in the present study. Bioinformatics analysis revealed that TRERNA1 may regulate Snail expression via endogenous competitive effects in combination with miR-1207-5p. However, it is necessary to further investigate how TRERNA1 regulates Snail expression via binding to miR-1270-5p in subsequent experiments.
At present, only a few studies have evaluated the prognostic value of TRERNA1 expression and suggested that TRERNA1 may act as a prognostic factor in gastric cancer $(35,36)$. TRERNA1 has been demonstrated to serve as an onco-lncRNA and to promote the metastasis and invasion of gastric cancer cells (35). In the present study, TRERNA1 expression was associated with the invasion and metastasis of CRC cells. Multivariate Cox regression model analysis was conducted to further assess the association between TRERNA1 expression and OS time in patients with CRC. The current results indicate that high TRERNA1 expression may serve as an independent predictor of poor prognosis in patients with $\mathrm{CRC}$, and that TRERNA1 may therefore be used as a prognostic marker in patients with CRC.

In conclusion, the present study was, to the best of our knowledge, the first to provide evidence that TRERNA1 was upregulated in CRC tissues compared with paired normal tissues. High TRERNA1 expression was positively associated with distant metastasis, perineural invasion, node metastasis stage, tumor diameter and OS time in patients with CRC. Knockdown of TRERNA1 inhibited the invasion and metastasis of CRC cells. However, extensive functional studies and additional well-designed studies with different ethnic groups are required to confirm the role of TRERNA1 in CRC prognosis.

\section{Acknowledgements}

Not applicable.

\section{Funding}

The present study was supported by grants from the Key Research and Development Foundation of Shandong Province (grant nos. 2017GSF218034, 2016GSF201010 and 2019GSF108016), the Science Foundation of Qilu Hospital of Shandong University (grant no. 2017QLQN16) and the China Postdoctoral Science Foundation (grant no. 2019M652393).

\section{Availability of data and materials}

The datasets used and/or analyzed during the present study are available from the corresponding author on reasonable request.

\section{Authors' contributions}

HQ and QH conceived the study; HQ and WW designed the experiments; WW performed the experiments; WW, XT and HQ analyzed the data and wrote the paper. All authors have read and approved the manuscript.

\section{Ethics approval and consent to participate}

The present study was approved by the Ethics Committee of Qilu Hospital of Shandong University (Jinan, China). Written informed consent was provided by all participants.

\section{Patient consent for publication}

Not applicable. 


\section{Competing interests}

The authors declare that they have no competing interests.

\section{References}

1. Jeun M, Lee HJ, Park S, Do EJ, Choi J, Sung YN, Hong SM, Kim SY, Kim DH, Kang JY, et al: A novel blood-based colorectal cancer diagnostic technology using electrical detection of colon cancer secreted protein-2. Adv Sci (Weinh) 6: 1802115, 2019

2. Chen W, Zheng R, Baade PD, Zhang S, Zeng H, Bray F, Jemal A, Yu XQ and He J: Cancer statistics in China, 2015. CA Cancer J Clin 66: 115-132, 2016.

3. Marisa L, Svrcek M, Collura A, Becht E, Cervera P, Wanherdrick K, Buhard O, Goloudina A, Jonchère V, Selves J, et al: The balance between cytotoxic T-cell lymphocytes and immune checkpoint expression in the prognosis of colon tumors. J Natl Cancer Inst 110, 2018.

4. van Bakel H, Nislow C, Blencowe BJ and Hughes TR: Most 'dark matter' transcripts are associated with known genes. PLoS Biol 8: e1000371, 2010.

5. Perkel JM: Visiting 'noncodarnia'. Biotechniques 54: 301, 303-304, 2013

6. Hu Q, Ye Y, Chan LC, Li Y, Liang K, Lin A, Egranov SD, Zhang Y, $\mathrm{Xia} \mathrm{W}$, Gong J, et al: Oncogenic lncRNA downregulates cancer cell antigen presentation and intrinsic tumor suppression. Nat Immunol 20: 835-851, 2019.

7. Ponting CP, Oliver PL and Reik W: Evolution and functions of long noncoding RNAs. Cell 136: 629-641, 2009.

8. Esteller M: Non-coding RNAs in human disease. Nat Rev Genet 12: 861-874, 2011.

9. Taft RJ,Pang KC, Mercer TR, Dinger M and Mattick JS: Non-coding RNAs: Regulators of disease. J Pathol 220: 126-139, 2010.

10. de Oliveira JC, Oliveira LC, Mathias C, Pedroso GA, Lemos DS Salviano-Silva A, Jucoski TS, Lobo-Alves SC, Zambalde EP, Cipolla GA and Gradia DF: Long non-coding RNAs in cancer: Another layer of complexity. J Gene Med 21: e3065, 2019.

11. Gupta RA, Shah N, Wang KC, Kim J, Horlings HM, Wong DJ, Tsai MC, Hung T, Argani P, Rinn JL, et al: Long non-coding RNA HOTAIR reprograms chromatin state to promote cancer metastasis. Nature 464: 1071-1076, 2010.

12. Ma Y, Yang Y, Wang F, Moyer MP, Wei Q, Zhang P, Yang Z, Liu W, Zhang $\mathrm{H}$, Chen $\mathrm{N}$, et al: Long non-coding RNA CCAL regulates colorectal cancer progression by activating Wnt/ $\beta$-catenin signalling pathway via suppression of activator protein $2 \alpha$. Gut 65: 1494-1504, 2016.

13. Neve B, Jonckheere N, Vincent A and Van Seuningen I: Epigenetic regulation by lncRNAs: An overview focused on UCA1 in colorectal cancer. Cancers (Basel) 10: pii: E440, 2018

14. Yang Y, Zhao L, Lei L, Lau WB, Lau B, Yang Q, Le X, Yang H, Wang C, Luo Z, et al: LncRNAs: The bridge linking RNA and colorectal cancer. Oncotarget 8: 12517-12532, 2017.

15. Chen DL, Lu YX, Zhang JX, Wei XL, Wang F, Zeng ZL, Pan ZZ, Yuan YF, Wang FH, Pelicano $\mathrm{H}$, et al: Long non-coding RNA UICLM promotes colorectal cancer liver metastasis by acting as a ceRNA for microRNA-215 to regulate ZEB2 expression. Theranostics 7: 4836-4849, 2017.

16. Han P, Li JW, Zhang BM, Lv JC, Li YM, Gu XY, Yu ZW, Jia YH, Bai XF, Li L, et al: The lncRNA CRNDE promotes colorectal cancer cell proliferation and chemoresistance via miR-181a-5p-mediated regulation of $\mathrm{Wnt} / \beta$-catenin signaling. Mol Cancer 16: 9, 2017.

17. Liu T, Han Z, Li H, Zhu Y, Sun Z and Zhu A: LncRNA DLEU1 contributes to colorectal cancer progression via activation of KPNA3. Mol Cancer 17: 118, 2018.

18. Ling H, Spizzo R, Atlasi Y, Nicoloso M, Shimizu M, Redis RS, Nishida N, Gafà R, Song J, Guo Z, et al: CCAT2, a novel noncoding RNA mapping to $8 \mathrm{q} 24$, underlies metastatic progression and chromosomal instability in colon cancer. Genome Res 23: 1446-1461, 2013

19. Wu Y, Yang X, Chen Z, Tian L, Jiang G, Chen F, Li J, An P, Lu L, Luo $\mathrm{N}$, et al: $\mathrm{m}^{6} \mathrm{~A}$-induced lncRNA RP11 triggers the dissemination of colorectal cancer cells via upregulation of Zeb1. Mol Cancer 18: 87, 2019.

20. Beyes S, Andrieux G, Schrempp M, Aicher D, Wenzel J, Antón-García P, Boerries M and Hecht A: Genome-wide mapping of DNA-binding sites identifies stemness-related genes as directly repressed targets of SNAIL1 in colorectal cancer cells. Oncogene 38: 6647-6661, 2019.
21. Wang H, Li JM, Wei W, Yang R, Chen D, Ma XD, Jiang GM and Wang BL: Regulation of ATP-binding cassette subfamily B member 1 by Snail contributes to chemoresistance in colorectal cancer. Cancer Sci 111: 84-97, 2020.

22. Peinado H, Olmeda D and Cano A: Snail, Zeb and bHLH factors in tumour progression: An alliance against the epithelial phenotype? Nat Rev Cancer 7: 415-428, 2007.

23. Ye X, Tam WL, Shibue T, Kaygusuz Y, Reinhardt F, Ng Eaton E and Weinberg RA: Distinct EMT programs control normal mammary stem cells and tumour-initiating cells. Nature 525: 256-260, 2015

24. Ye C, Shen Z, Wang B, Li Y, Li T, Yang Y, Jiang K, Ye Y and Wang S: A novel long non-coding RNA lnc-GNAT1-1 is low expressed in colorectal cancer and acts as a tumor suppressor through regulating RKIP-NF- $\kappa$ B-Snail circuit. J Exp Clin Cancer Res 35: 187, 2016.

25. Yang MH, Hu ZY, Xu C, Xie LY, Wang XY, Chen SY and Li ZG: MALAT1 promotes colorectal cancer cell proliferation/migration/invasion via PRKA kinase anchor protein 9. Biochim Biophys Acta 1852: 166-174, 2015.

26. Ji Q, Zhang L, Liu X, Zhou L, Wang W, Han Z, Sui H, Tang Y, Wang Y, Liu N, et al: Long non-coding RNA MALAT1 promotes tumour growth and metastasis in colorectal cancer through binding to SFPQ and releasing oncogene PTBP2 from SFPQ/PTBP2 complex. Br J Cancer 111: 736-748, 2014

27. Ji Q, Liu X, Fu X, Zhang L, Sui H, Zhou L, Sun J, Cai J, Qin J, Ren $\mathbf{J}$ and Li Q: Resveratrol inhibits invasion and metastasis of colorectal cancer cells via MALAT1 mediated Wnt/ $\beta$-catenin signal pathway. PLoS One 8: e78700, 2013.

28. Shen X, Bai Y, Luo B and Zhou X: Upregulation of IncRNA BANCR associated with the lymph node metastasis and poor prognosis in colorectal cancer. Biol Res 50: 32, 2017.

29. Ragusa M, Barbagallo C, Statello L, Condorelli AG, Battaglia R, Tamburello L, Barbagallo D, Di Pietro C and Purrello M: Non-coding landscapes of colorectal cancer. World J Gastroenterol 21: 11709-11739, 2015.

30. Zhang Z, Jia H, Gu T, Hu Q, Yu J, Zang D, Song N and Wang H: RNA sequencing and bioinformatics analysis of the long noncoding RNA-mRNA network in colorectal cancer. J Cell Biochem 119: 9957-9966, 2018

31. Edge SB and Compton CC: The American joint committee on cancer: The 7th edition of the AJCC cancer staging manual and the future of TNM. Ann Surg Oncol 17: 1471-1474, 2010.

32. Patel KR, Andreadi C, Britton RG, Horner-Glister E, Karmokar A, Sale S, Brown VA, Brenner DE, Singh R, Steward WP, et al: Sulfate metabolites provide an intracellular pool for resveratrol generation and induce autophagy with senescence. Sci Transl Med 5: 205ra133, 2013.

33. Livak KJ and Schmittgen TD: Analysis of relative gene expression data using real-time quantitative PCR and the 2(-Delta Delta C(T)) method. Methods 25: 402-408, 2001.

34. Enright AJ, John B, Gaul U, Tuschl T, Sander C and Marks DS: MicroRNA targets in Drosophila. Genome Biol 5: R1, 2003.

35. Wu H, Hu Y, Liu X, Song W, Gong P, Zhang K, Chen Z, Zhou M, Shen X, Qian Y and Fan H: LncRNA TRERNA1 function as an enhancer of SNAI1 promotes gastric cancer metastasis by regulating epithelial-mesenchymal transition. Mol Ther Nucleic Acids 8: 291-299, 2017.

36. Wu H, Liu X, Gong P, Song W, Zhou M, Li Y, Zhao Z and Fan H: Elevated TFAP4 regulates IncRNA TRERNA1 to promote cell migration and invasion in gastric cancer. Oncol Rep 40: 923-931, 2018.

37. Song W, Gu Y, Lu S, Wu H, Cheng Z, Hu J, Qian Y, Zheng Y and Fan H: LncRNA TRERNA1 facilitates hepatocellular carcinoma metastasis by dimethylating $\mathrm{H} 3 \mathrm{~K} 9$ in the $\mathrm{CDH} 1$ promoter region via the recruitment of the EHMT2/SNAI1 complex. Cell Prolif 52: e12621, 2019.

38. Wang X and Wu X: The role of MicroRNA-1207-5p in colorectal cancer. Clin Lab 63: 1875-1882, 2017.

39. Yan Y, Su M and Qin B: CircHIPK3 promotes colorectal cancer cells proliferation and metastasis via modulating of miR-1207-5p/FMNL2 signal. Biochem Biophys Res Commun 524: 839-846, 2020.

40. Ma S, Yang D, Liu Y, Wang Y, Lin T, Li Y, Yang S, Zhang W and Zhang R: LncRNA BANCR promotes tumorigenesis and enhances adriamycin resistance in colorectal cancer. Aging (Albany NY) 10: 2062-2078, 2018.

41. Li AX, Xin WQ and Ma CG: Fentanyl inhibits the invasion and migration of colorectal cancer cells via inhibiting the negative regulation of Ets-1 on BANCR. Biochem Biophys Res Commun 465: 594-600, 2015. 
42. Xie X, Tang B, Xiao YF, Xie R, Li BS, Dong H, Zhou JY and Yang SM: Long non-coding RNAs in colorectal cancer. Oncotarget 7: 5226-5239, 2016.

43. Nissan A, Stojadinovic A, Mitrani-Rosenbaum S, Halle D, Grinbaum R, Roistacher M, Bochem A, Dayanc BE, Ritter G, Gomceli I, et al: Colon cancer associated transcript-1: A novel RNA expressed in malignant and pre-malignant human tissues. Int J Cancer 130: 1598-1606, 2012.

44. Kim T, Jeon YJ, Cui R, Lee JH, Peng Y, Kim SH, Tili E, Alder H and Croce CM: Role of MYC-regulated long noncoding RNAs in cell cycle regulation and tumorigenesis. J Natl Cancer Inst 107: pii: dju505, 2015.

45. Miller CR, Ruppert AS, Fobare S, Chen TL, Liu C, Lehman A, Blachly JS, Zhang X, Lucas DM, Grever MR, et al: The long noncoding RNA, treRNA, decreases DNA damage and is associated with poor response to chemotherapy in chronic lymphocytic leukemia. Oncotarget 8: 25942-25954, 2017.

46. Yang YR, Jang HJ, Yoon S, Lee YH, Nam D, Kim IS, Lee H, Kim H, Choi JH, Kang BH, et al: OGA heterozygosity suppresses intestinal tumorigenesis in Apc(min/+) mice. Oncogenesis 3: e109, 2014
47. Barrallo-Gimeno A and Nieto MA: The Snail genes as inducers of cell movement and survival: Implications in development and cancer. Development 132: 3151-3161, 2005.

48. Cano A, Pérez-Moreno MA, Rodrigo I, Locascio A, Blanco MJ, del Barrio MG, Portillo F and Nieto MA: The transcription factor snail controls epithelial-mesenchymal transitions by repressing E-cadherin expression. Nat Cell Biol 2: 76-83, 2000.

49. Batlle E, Sancho E, Francí C, Domínguez D, Monfar M, Baulida J and García De Herreros A: The transcription factor snail is a repressor of E-cadherin gene expression in epithelial tumour cells. Nat Cell Biol 2: 84-89, 2000.

(i) (2) This work is licensed under a Creative Commons Attribution-NonCommercial-NoDerivatives 4.0 International (CC BY-NC-ND 4.0) License. 